Methods A non-inferiority, open-label, single center RCT was conducted in Prague, Czech Republic. Patients, 18-75 years of age, diagnosed with uncomplicated rectal or pharyngeal gonorrhea by nucleic acid amplification test (NAAT) (GeneProof ${ }^{\mathbb{B}}$ ) were randomized to treatment with gentamicin $240 \mathrm{mg}$ intramuscularly plus azithromycin $2 \mathrm{~g}$ orally or ceftriaxone $500 \mathrm{~g}$ intramuscularly plus azithromycin $2 \mathrm{~g}$ orally. The primary outcome was negative culture and negative NAAT, i.e., one week and three weeks, respectively, after treatment.

Results Both clinical and microbiological cure was achieved by $100 \%$ of patients in the gentamicin +azithromycin arm $(n=68$; 40 rectal, 14 pharyngeal, and 14 infections in both localizations) and ceftriaxone+azithromycin arm ( $\mathrm{n}=66 ; 36$ rectal, 14 pharyngeal, and 16 infections in both localizations). Administration of gentamicin was significantly less painful than ceftriaxone according to the visual analog score $(\mathrm{p}<0.001)$. Gastrointestinal adverse events were slightly more common in ceftriaxone arm (50.0\%) than in gentamicin arm (41.2\%), but in most (64\%) cases they were mild.

Conclusion Both gentamicin+azithromycin and ceftriaxone +azithromycin were $100 \%$ effective for treatment of rectal and pharyngeal gonorrhea. Gentamicin $240 \mathrm{mg}$ plus azithromycin $2 \mathrm{~g}$ appears to be an effective alternative for treatment of both urogenital and extragenital gonorrhea in case of ceftriaxone resistance, allergy, or unavailability.

Disclosure No significant relationships.

\section{P651 ELUCIDATING THE EFFECT OF ESCULETIN AGAINST GLUTAMATE RACEMASE - A NOVEL DRUG TARGET OF NEISSERIA GONORRHOEAE}

${ }^{1}$ Alka Pawar*, ${ }^{1}$ Chandrika Konwar, ${ }^{1}$ Prakash tha ${ }^{1}$ Madhu Chopra, ${ }^{2}$ Uma Chaudhry, 'Daman Saluja. 'University of Delhi, New Delhi, Dr. B R Ambedkar Center for Biomedical Research (ACBR), New Delhi, India; '2Bhaskeracharya College of Applied Sciences, Biomedical Research, Delhi, India

10.1136/sextrans-2019-sti.719

Background Neisseria gonorrhoeae (NG) is a sexually transmitted pathogen infecting both men and women. In spite of a number of antibiotics, gonorrhea (also known as "The Clap"), remains a frequently reported STI and is an important cause of pelvic inflammatory disease and infertility. Due to resistance to most of the currently used drugs, $N G$ has been named as 'Superbug' posing a serious threat to gonorrhoea treatment worldwide. Therefore, there is an urgent need to find novel drug targets and to develop new antibacterial agents.

Methods Using system biology to identify potential drug targets and the known inhibitors/drugs against homologous proteins, we identified a novel drug target, namely glutamate racemase (GR). This enzyme is involved in the early phase of peptidoglycan biosynthesis in both gram positive and gram negative bacteria. As protein-ligand interactions play a key role in structure-based drug design, we screened natural compounds for binding to NG-GR by carrying out docking studies, shortlisted the best docked compounds and evaluated them for their functional, structural and antibacterial activity.

Results The computational analysis showed that the coumarin derivative-esculetin exhibited best binding affinity among all the tested compounds. Characterization of the biophysical properties of purified recombinant GR using circular dichroism, in the absence and presence of esculetin, indicated a change in protein conformation in the presence of esculetin. This change is the protein structure was associated with a concomitant inhibition of racemization activity of recombinant GR. Esculetin also inhibited the growth of the bacteria in culture both in time and concentration dependent manner.

Conclusion In conclusion, these observations could provide impetus for further research in this direction. Better understanding of antibacterial mechanisms of esculetin will help in establishing lead molecules for the treatment of gonococcal infections.

Disclosure No significant relationships.

\section{P652 USE OF SPECIMENS SUBMITTED FOR NEISSERIA GONORRHOEAE MOLECULAR TESTING TO ENHANCE SURVEILLANCE IN A CANADIAN ARCTIC TERRITORY}

${ }^{1}$ Kethika Kulleperuma, ${ }^{1}$ Sonia Marchand, ${ }^{2}$ rene Martin, ${ }^{1}$ Jasmine Pawa, ${ }^{3}$ Ameeta Singh* ${ }^{1}$ Government of Nunavut, Iqaluit, Canada; ${ }^{2}$ National Microbiology Laboratory, Winnipeg, Canada; ${ }^{3}$ University of Alberta, Medicine/Infectious Diseases, Edmonton, Canada

\subsection{6/sextrans-2019-sti. 720}

Background Surveillance for antimicrobial resistance (AMR) in Neisseria gonorrhoeae (NG) is critical to monitor trends and to inform treatment guidelines for NG. In Nunavut (NU), a Canadian Arctic territory, culture for NG is not possible due to environmental conditions which affect organism viability. Specimens submitted for nucleic acid amplification tests (NAAT) are routinely used to screen for NG and have the potential to provide important surveillance information with additional testing.

Methods In January, 2018, Nunavut commenced submission of Roche-Cobas NG positive NAAT specimens to the National Microbiology Laboratory (Winnipeg, Canada). Samples were sequenced using Neisseria gonorrhoeae multi-antigen sequence typing (NG-MAST). Real-time (RT-) PCR assays were used to detect single nucleotide polymorphisms (SNPs) in genes associated with ciprofloxacin resistance (gyrA, parC) and azithromycin resistance (23S rRNA).

Results From January to September, 2018, 257 samples were submitted to NML. 229 samples were typeable and 21 different NG-MAST STs were identified, of which approximately half are unique to NU. The most prevalent ST was ST16840 $(34.5 \%, 79 / 229)$ which is associated with ciprofloxacin resistance and is closely related to ST 10451, a common sequence type identified across Canada. 28.5\% (66/229) were ST5985, a common ST circulating in Canada and is associated with tetracycline resistance. Fully susceptible ST 4637 represented $10.5 \%(24 / 229)$ specimens. Of 216 specimens, 92 were predicted to be ciprofloxacin resistant and of 218 specimens; less than five samples were predicted to be azithromycin resistant. Conclusion Data from Nunavut, a Canadian Arctic region where the collection of NG culture is not feasible, support the use of NAAT positive NG specimens to provide enhanced surveillance to monitor the types of NG circulating in a community and ensure that currently recommended therapies are appropriate. Future surveillance initiatives linking the samples to epidemiologic data has the potential to support additional public health interventions in at-risk populations.

Disclosure No significant relationships. 